\title{
Pilot contamination reduction in massive MIMO systems based on pilot scheduling
}

\author{
Yucheng $\mathrm{Wu}^{*}{ }^{\dagger}$, Tong Liu, Meng Cao, Liang Li and Weiyang $\mathrm{Xu}^{\dagger}$
}

\begin{abstract}
The pilot contamination caused by sharing the non-orthogonal pilots among users is considered to be a bottleneck of the massive multi-input multi-output (MIMO) systems. This paper proposes a pilot scheduling scheme based on the degradation to address this problem. Through computing the degradation of the users, the proposed scheduling assigns the optimal pilot sequence to the user who suffers from the greatest degradation in a greedy way. Moreover, the proposed scheme is further optimized with an extra set of orthogonal pilot sequences, which is called pilot scheduling scheme based on user grouping. Simulation results show that the target cell's achievable sum rate of the proposed scheme is much higher than the random pilot scheduling (RPS) and the smart pilot assignment (SPA) schemes; also, our scheme can reduce the impact of shadowing fading on the target cell's achievable sum rate effectively.
\end{abstract}

Keywords: Massive MIMO, Pilot contamination, Pilot scheduling, Greedy algorithm, User grouping

\section{Introduction}

Massive multi-input multi-output (MIMO) [1-3] is one of the key technologies in $5 \mathrm{G}$, which can greatly boost the channel capacity, spectral efficiency, and connection density by utilizing a large number of antennas at the base station (BS). For a short coherence time, the same pilot sequences are usually assigned to users in different cells to save bandwidth, leading to the problem of pilot contamination [4]. When the number of BS antennas goes to infinity, the system capacity is largely limited by the inter-cell interference because of the pilot contamination [5]. Therefore, pilot pollution has become one of the main reasons for the performance loss in massive MIMO systems.

Currently, pilot contamination reduction schemes in massive MIMO systems can be classified into three groups: channel estimation [6-11], precoding [12], and pilot scheduling [13-20]. In [6], pilot contamination was tackled by a multi-cell precoding method based on the minimum mean square error channel estimation. Under the assumption of spatially correlated channels, it has been found that the pilot contamination would disappear

\footnotetext{
*Correspondence: wuyucheng@cqu.edu.cn

${ }^{\dagger}$ Equal contributors

${ }^{1}$ College of Communication Engineering, Chongqing University, No.174

Shazhengjie, Chongqing, China
}

by exploiting the covariance information of user channels and using a coordinated pilot assignment strategy among cells [7]. Alternatively, the concept of time-shifted pilots was proposed to avoid the inter-cell interference via rearranging the order of uplink pilot transmission in different cells [8]. However, this asynchronous transmission scheme may result in strong interference when two closely located users in neighboring cells are transmitting or receiving simultaneously [9]. The sparse Bayesian learning was employed to estimate not only the CSI but also the interference from neighboring cells [10]. A compressive sensing-based low-rank approximation scheme for channel estimation was proposed in [11], which exploits the fact that the degree of freedom of physical channels is much smaller than the number of independent parameters.

Specifically, this manuscript focuses on the pilot scheduling because in essence, pilot contamination is incurred by the assignment of the same pilot sequences to users in neighboring cells [13]. Therefore, pilot scheduling considers how the system reassigns the pilot sequences to users so that the pilot contamination problem can be alleviated or even eliminated. In general, pilot scheduling schemes include time-shifted pilots, power control, fractional pilot reuse, and pilot coordination scheduling. 
To avoid the inter-cell interference, the concept of timeshifted pilots was proposed to rearrange the order of uplink pilot transmission in different cells in the frame structure [14]. However, this asynchronous transmission scheme may result in strong interference when two closely located users in adjacent cells are transmitting or receiving simultaneously.

In power control method, the transmit power is controlled so that the user groups with large cross gains choose different transmission time slots or the transmit power of users assigned with the same pilot sequences is reduced [15]. Unfortunately, this method needs a control mechanism to make sure the pilot sequences are synchronous in adjacent cells; thus, it becomes inefficient or even inapplicable when the number of antennas at the BS grows large. In [16], the authors developed techniques based on existing long-term evolution measurementsopen loop power control and pilot sequence reuse schemes, that avoid pilot contamination within a group of cell. While in [17], a power control problem to minimize D2D links' data transmit power is formulated and an iterative scheme is adopted to solve the problem.

Fractional pilot reuse method introduces an extra pilot overhead to reduce the pilot contamination. Generally, this method can be classified into two categories. The first divides the cells into two groups. The cells in the same group are assigned with the same pilot sequences, but those in different cells are assigned with the orthogonal pilot sequences in order to mitigate the pilot contamination $[18,19]$. The second category divides the users into two groups according to different levels of pilot contamination, namely the center users who suffer from the modest pilot contamination and the edge users who suffer from severe pilot contamination. A cell-center pilot group is reused for all cell-center users, while a cell-edge pilot group is applied for the edge users in the adjacent cells [20]. This method improves the quality of service (QoS) of the edge users at the cost of a slight rate loss of the center users.

Pilot coordination scheduling scheme reassigns the pilot sequences to users according to different levels of QoS. However, the cooperation among cells incurs an extra computational burden. The pilot coordination scheduling scheme includes pilot scheduling based on maximum posteriori estimation [21], smart pilot assignment (SPA) [22], and pilot scheduling based on greedy algorithm [23].

To obtain the maximum of the target cell's achievable sum rate, exhaustive search is a simple way to find the optimal solution [24]. However, this method is of great complexity and limited by the system coherence time; thus, the random pilot scheduling (RPS) schemes are usually chosen instead [4]. As an alternative, SPA scheme aims to ensure fairness among all users in the target cell by maximizing the minimum uplink signal-to- interference plus noise ratio (SINR) [22]. Both RPS and SPA do not take into account the users' uplink requirement, leading to a rate loss in the target cell's achievable sum rate that users cannot meet the requirement of QoS adequately. To solve this problem, a pilot scheduling scheme based on the performance degradation is proposed in this paper. Specifically, the proposed scheme assigns the optimal pilot sequence to the user who suffers from the greatest degradation greedily by computing the performance degradation of all users. However, users with poor channel condition may have performance bottleneck problem, causing communication interrupted. Therefore, a pilot scheduling scheme based on user grouping according to different levels of pilot contamination is introduced when an extra set of orthogonal pilot sequences is available.

The rest of the paper is organized as follows. In Section 2, we briefly review the massive MIMO multicell multi-user system model, while Section 3 is devoted to detailing the pilot contamination, which is the main performance-limiting factor of massive MIMO systems. The proposed schemes, namely the pilot scheduling schemes based on performance degradation and user grouping, are discussed in Section 4. Our simulation results quantifying the benefits of our proposals are presented in Section 5, while the conclusions follow in Section 6.

Notation: $\mathbb{C}$ indicates the set of complex numbers. Bold font variables represent matrices and vectors. $(\cdot)^{T}$ and $(\cdot)^{H}$ denote the transpose and complex conjugate transpose, separately. $[\mathbf{A}]_{m n}$ is the element on the $m$ th row and $n$th column of a matrix A. $\mathbf{I}_{M}$ indicates an identity matrix of size $M \times M$. For a random vector $\mathbf{x}, \mathbf{x} \sim \mathcal{C} N(\boldsymbol{\mu}, \boldsymbol{\sigma})$ means it follows a complex Gaussian distribution with mean $\boldsymbol{\mu}$ and covariance $\boldsymbol{\sigma}$. Finally, $\mathbb{E}\{\cdot\}$ represents the expectation operator.

\section{Methods}

This study originated from a need to reduce the pilot contamination in massive MIMO systems. A scenario of multi-cell multi-user massive MIMO, where the uplink transmit power is assumed to be equal for all users is considered. Specifically, the proposed scheme reduces the pilot contamination through pilot scheduling, based on the observation that different pilots contribute differently to the amount of interference. First, the proposed scheduling assigns the optimal pilot sequence to the user suffering from the greatest degradation via computing the degradation of the users. Second, the proposed scheme is further optimized with a pilot scheduling scheme based on user grouping. Based on the proposed algorithm, numerical simulation is conducted taking the achievable rate of target cell into consideration. Simulation results show that the proposed scheme achieves a higher rate than RPS 
and SPA methods on the same conditions; also, it can reduce the impact of shadowing fading on the target cell's achievable sum rate effectively.

\section{System model}

A multi-cell multi-user massive MIMO system is illustrated in Fig. 1, with each hexagonal cell having a central BS associated with $M$ antennas to serve $K(K \gg M)$ single-antenna users. The channel vector $h_{i j k} \in \mathbb{C}^{M \times 1}$ denotes the link spanning from the $k$ th user of the $j$ th cell to the BS of the $i$ th cell, which can be formulated as

$$
h_{i j k}=g_{i j k} \sqrt{\beta_{i j k}} .
$$

The small-scale fading vectors $g_{i j k} \in \mathbb{C}^{M \times 1}$ are statistically independent for the $K$ users and complex Gaussiandistributed with a zero-mean vector and a covariance matrix $\mathbf{I}_{M}$, i.e., $g_{i j k} \in \mathcal{C} N\left(\mathbf{0}, \mathbf{I}_{M}\right)$. Still referring to (1), the large-scale fading coefficients $\beta_{i j k}$ are the same for the different antennas at the same BS, but they are userdependent. Moreover, they relate to both the path loss and shadow fading, which can be modeled as $\beta_{i j k}=\frac{Z_{i j k}}{\left(r_{i j k} / R\right)^{\alpha}}$, where $Z_{i j k}$ represents the shadow fading, $r_{i j k}$ is the distance between the $k$ th user in the $j$ th cell and the BS in the $i$ th cell, and $R$ is the cell radius. Thus, the matrix modeling the channel between all the $K$ users in the $j$ th cell and the BS in the $j$ th cell can be denoted by

$$
\mathbf{H}_{j l}=\mathbf{G}_{j l} \mathbf{D}_{j l}^{\frac{1}{2}}
$$

where $\mathbf{G}_{j l} \in \mathbb{C}^{M \times K}$ is the matrix of fast-fading coefficients between the $M$ antennas of the BS in the $l$ th cell and $K$ users in the $j$ th cell, and $\mathbf{D}_{j l} \in \mathbb{C}^{K \times K}$ is a diagonal matrix whose diagonal elements are $\left[\mathbf{D}_{j l}\right]_{k k}=\beta_{j l k}$. Furthermore, the channel vectors are assumed to be constant during the coherence time and change independently in different coherence intervals.

\section{Pilot contamination}

In multi-cell multi-user massive MIMO systems, the BSs obtain the downlink channel estimate by exploiting the uplink pilot sequence within a coherence time. At the duration of data transmission, all users synchronously send uplink data to their corresponding BSs and the user data received at the $\mathrm{BS}$ in the $i$ th cell can be denoted as

$$
\boldsymbol{y}_{i}^{u}=\sqrt{P_{u}} \sum_{j=1}^{L} \sum_{k=1}^{K} h_{i j k} x_{j k}^{u}+\boldsymbol{n}_{i}^{u},
$$

where $x_{j k}^{u}$ with $\mathbb{E}\left\{\left|x_{j k}^{u}\right|^{2}\right\}=1$ denotes the symbol transmitted from the $k$ th user roaming in the $j$ th cell, $P_{u}$ presents the uplink transmit power, and $n_{i}^{u} \in \mathbb{C}^{M \times 1}$ denotes the additive white Gaussian noise (AWGN) vector associated with $\mathbb{E}\left\{\boldsymbol{n}_{i}^{u}\left(\boldsymbol{n}_{i}^{u}\right)^{H}\right\}=\sigma_{n}^{2} \mathbf{I}_{M}$.

On the other hand, all users transmit uplink pilot sequences to BSs simultaneously at the duration of pilot transmission. For a typical massive MIMO system, the pilot sequences used within a specific cell are orthogonal, but the same pilot group is typically reused in the adjacent cells due to the limited number of orthogonal pilot sequences. Thus, the matrix of pilot sequences received at the BS of the $i$ th cell can be denoted as

$$
\boldsymbol{y}_{i}^{p}=\sqrt{P_{p}}\left(\sum_{j=1}^{L} \mathbf{H}_{i j} \boldsymbol{\Phi}_{i}\right)+\boldsymbol{n}_{i}^{p},
$$

where $y_{i}^{p} \in \mathbb{C}^{M \times \tau}$ with $\tau$ being the time duration of pilot transmission, and the matrix $\boldsymbol{\Phi}_{i}=\left(\phi_{1}, \ldots, \phi_{K}\right)^{T}$ contains the transmitted pilot sequences satisfying the power constraint $\boldsymbol{\Phi}_{i} \boldsymbol{\Phi}_{i}^{H}=\mathbf{I}_{K} . P_{p}$ is the transmit power of the pilots, and $\boldsymbol{n}_{i}^{p} \in \mathbb{C}^{M \times \tau}$ denotes the uplink channel's noise vector.

The BS of the $i$ th cell obtains an estimate of the channel matrix $\mathbf{H}_{i i}$ using any conventional channel estimation methods, for instance, the least square (LS) approach

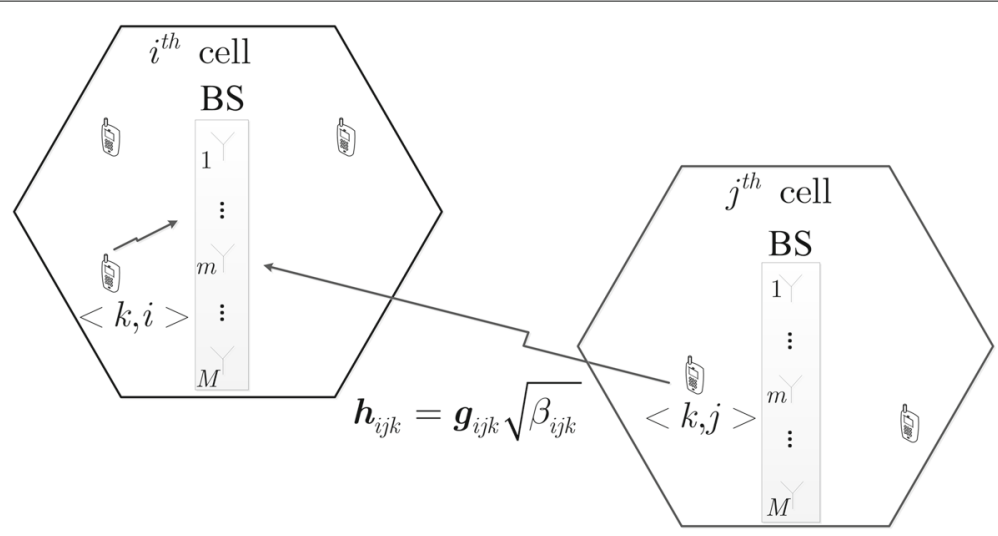

Fig. 1 The model of a massive MIMO multi-cell multi-user system 
which directly correlates the received pilot matrix with the local pilot matrix, i.e.,

$$
\hat{\mathbf{H}}_{i i}=\frac{1}{\sqrt{P_{p}}} \boldsymbol{y}_{i}^{p} \boldsymbol{\Phi}_{i}^{H}=\mathbf{H}_{i i}+\sum_{j \neq i} \mathbf{H}_{i j}+\frac{1}{\sqrt{P_{p}}} \boldsymbol{n}_{i}^{p} \boldsymbol{\Phi}_{i}^{H} .
$$

It can be readily seen that the channel estimate of the $k$ th user in the $i$ th cell, namely $\hat{h}_{i i k}$, is a linear combination of the channels $h_{i j k}$ for $1 \leq j \leq L$, which includes the channels of the users in the other cells associated with the same pilot sequence. This phenomenon is referred to as pilot contamination. Given the estimated channel matrix $\hat{\mathbf{H}}_{i i}$ and by adopting the low-complexity match filter (MF) detector, the detected symbol arriving from the $k$ th user in the $i$ th cell can be denoted as

$$
\begin{aligned}
\hat{x}_{i k}^{u} & =h_{i i k}^{H} y_{i}^{u} \\
& =\left(\sum_{j=1}^{L} h_{i j k}^{H}+v_{i k}^{H}\right)\left(\sqrt{P_{u}} \sum_{j=1}^{L} \sum_{k=1}^{K} h_{i j k} x_{j k}^{u}+n_{i}^{u}\right) \\
& =\sum_{j=1}^{L} h_{i j k}^{H} \cdot \sqrt{P_{u}} \sum_{j=1}^{L} \sum_{k=1}^{K} h_{i j k} x_{j k}^{u}+\varepsilon_{i k}^{u} \\
& =\sqrt{P_{u}}\left(h_{i i k}^{H} h_{i i k} x_{i k}^{u}+\sum_{j \neq i} h_{i j k}^{H} h_{i j k} x_{j k}^{u}\right)+\varepsilon_{i k}^{u} \\
& =M \sqrt{P_{u}}\left(\beta_{i i k} x_{i k}^{u}+\sum_{j \neq i} \beta_{i j k} x_{j k}^{u}\right)+\varepsilon_{i k}^{u}
\end{aligned}
$$

where $v_{i k}$ denotes the $k$ th column of $\frac{n_{i}^{p} \Phi_{i}^{H}}{\sqrt{P_{p}}}$, where $\varepsilon_{i k}^{u}$ indicates the orthogonality between channels of different users, and it is well known that when the number of $M \rightarrow \infty$, it is almost surely that $\varepsilon_{i k}^{u} \rightarrow 0$. Therefore, when the number of BS antennas goes to infinity, we have

$$
\hat{x}_{i k}^{u} \approx M \sqrt{P_{u}}\left(\beta_{i i k} x_{i k}^{u}+\sum_{j \neq i} \beta_{i j k} x_{j k}^{u}\right) .
$$

Thus, the uplink SINR of the $k$ th user in the $i$ th cell can be calculated by

$$
\operatorname{SINR}_{i k}^{u}=\frac{\left|h_{i i k}^{H} h_{i i k}\right|^{2}}{\sum_{j \neq i}\left|h_{i j k}^{H} h_{i j k}\right|^{2}+\frac{\left|\varepsilon_{i k}^{u}\right|^{2}}{P_{u}}} .
$$
have

When the number of BS antennas goes to infinity, we

$$
\operatorname{SINR}_{i k}^{u}=\frac{\beta_{i i k}^{2}}{\sum_{j \neq i} \beta_{i j k}^{2}} .
$$

And the achievable uplink rate can be expressed as

$$
C_{i k}^{u}=\left(1-\mu_{0}\right) \mathbb{E}\left\{\log _{2}\left(1+\operatorname{SINR}_{i k}^{u}\right)\right\},
$$

where $0<\mu_{0}<1$ evaluates the spectral efficiency reduction caused by the pilot transmission. It is clear that the uplink achievable rate is limited by the pilot contamination and cannot be increased by simply assigning more transmit power.

\section{Proposed pilot scheduling scheme}

In this section, the proposed pilot scheduling schemes based on the performance degradation and user grouping will be discussed. First, we summarize the asymptotic orthogonality between random vectors. Let $\mathbf{x}, \mathbf{y} \in$ $\mathbb{C}^{M \times 1}$ be the two independent vectors with distribution $\mathcal{C} N\left(\mathbf{0}, c \mathbf{I}_{M}\right)$, and then

$$
\lim _{M \rightarrow \infty} \frac{\mathbf{x}^{\dagger} \mathbf{y}}{M} \stackrel{\text { a.s. }}{\longrightarrow} 0 \text { and } \lim _{M \rightarrow \infty} \frac{\mathbf{x}^{\dagger} \mathbf{x}}{M} \stackrel{\text { a.s. }}{\longrightarrow} c \text {, }
$$

where $\stackrel{\text { a.s. }}{\longrightarrow}$ denotes the almost sure convergence.

\subsection{Pilot scheduling scheme based on the degradation}

In this section, the pilot assignment for a target cell is firstly formulated as an optimization problem. Then, the pilot scheduling scheme based on the degradation is proposed to search for the optimization solution in a greedy way.

\subsubsection{Problem formulation}

First, we define a utility function which denotes the uplink achievable rate when the pilot sequence $\phi_{k}$ is assigned to the user $U_{k}$, i.e.,

$$
\Theta\left(U_{k}, \phi_{k}\right)=C_{U_{k}}^{u}\left(U_{k}, \phi_{k}\right),
$$

where $\left(U_{k}, \phi_{k}\right)$ means that the pilot sequence $\phi_{k}$ is assigned to the user $U_{k} .\left\{F_{s}: s=1, \ldots, K !\right\}$ denotes all $K$ ! possibilities of pilot assignments, e.g., $F_{s}=\left[f_{s}^{1}, f_{s}^{2}, \ldots, f_{s}^{K}\right]$ denotes the $s$ th assignment. We also assume that the pilot sequence $\phi_{k}$ is assigned to the $k$ th user $U_{k}$ in all other cells.

In massive MIMO systems, it is expected to maximize the target cell's achievable sum rate, which can be formulated as the following optimization problem

$$
P: \max _{\left\{F_{s}\right\}}\left\{\sum_{k=1}^{K} C_{U_{k}}^{u}\left(U_{k}, \phi_{f_{s}}\right)\right\} .
$$

According to (12), we have

$C_{U_{k}}^{u}\left(U_{k}, \phi_{f_{s}^{k}}\right)=\left\{\left(1-\mu_{0}\right) \log _{2}\left(1+\operatorname{SINR}_{u_{k}}^{u}\left(U_{k}, \phi_{f_{s}^{k}}\right)\right)\right\}$,

where $\operatorname{SINR}_{u_{k}}^{u}\left(U_{k}, \phi_{f_{s}^{k}}\right)$ denotes the SINR when the pilot sequence $\phi_{f_{s}^{k}}$ is assigned using the proposed algorithm to user $U_{k}$. According to (8), it comes to 


$$
\operatorname{SINR}_{u_{k}}^{u}\left(U_{k}, \phi_{f_{s}^{k}}\right)=\frac{\left|h_{i i k}^{H} h_{i i k}\right|^{2}}{\sum_{j \neq i}\left|h_{i j f_{s}^{k}}^{H} h_{i j f_{s}^{k}}\right|^{2}+\frac{\left|\varepsilon_{i k}^{u}\right|^{2}}{P_{u}}}
$$

However, it is impossible to solve this optimization problem because the accurate channel estimate under pilot contamination is not available. Fortunately, the upper bound of the uplink SINR can be calculated by the largescale fading coefficients $\beta_{i j k}$

$$
\operatorname{SINR}_{u_{k}}^{u}\left(U_{k}, \phi_{f_{s}^{k}}\right)=\frac{\beta_{i i k}^{2}}{\sum_{j \neq i} \beta_{i i f f_{s}^{k}}^{2}} .
$$

As we know, the large-scale fading coefficients $\beta_{i j k}$ changes slowly and can be easily tracked by the BSs. Thus, the optimization problem can be transformed into

$$
P: \max _{\left\{F_{s}\right\}}\left\{\left(1-\mu_{0}\right) \sum_{k=1}^{K} \log _{2}\left(1+\frac{\beta_{i i k}^{2}}{\sum_{j \neq i} \beta_{i i f_{s}^{k}}^{2}}\right)\right\} .
$$

The proposed scheme gives a solution to this optimization problem $P$ in a greedy way, which will be addressed in detail in the next subsection.

\subsubsection{Pilot assignment}

The proposed pilot assignment strategy maximizes the target cell's achievable sum rate based on performance degradation of users who have not been assigned with pilot sequences. We consider the pilot assignment in a specific cell, i.e., taking the $i$ th cell as the target cell, and the pilot assignment in other cells are independently managed by their corresponding BSs.

Let $U$ denote the set containing all users who have not been assigned with pilot sequences, $N_{U}$ denote the number of element in the set $U$, and $\Phi$ indicate the available pilot sequence set, while $N_{\Phi}$ is the number of element in the set $\Phi$. For user $U_{k} \in U$, we first compute its optimal pilot sequence, i.e., the pilot which can obtain the greatest utility function,

$$
\phi^{\mathrm{opt}}\left(U_{k}\right)=\arg \max _{\phi \in \Phi}\left\{\Theta\left(U_{k}, \phi_{1}\right), \cdots, \Theta\left(U_{k}, \phi_{N_{\Phi}}\right)\right\} .
$$

The suboptimal pilot sequence of user $U_{k}$ is defined as the pilot that can obtain the greatest utility function when every available pilot sequence except the optimal pilot sequence is assigned to the user, i.e.,

$$
\phi^{\mathrm{sub}}\left(U_{k}\right)=\arg \max _{\phi \in \Phi}\left\{\Theta\left(U_{k}, \phi_{1}\right) \backslash \Theta\left(U_{k}, \phi^{\mathrm{opt}}\left(U_{k}\right)\right)\right\} .
$$

Thus, the performance degradation of the user $U_{k}$ can be computed as follows:

$$
d\left(U_{k}\right)=\Theta\left(U_{k}, \phi^{\mathrm{opt}}\left(U_{k}\right)\right)-\Theta\left(U_{k}, \phi^{\mathrm{sub}}\left(U_{k}\right)\right) .
$$

Next, we compute the degradation of every user who has not ever been assigned with pilot sequences. Among these users, we find the user who suffers the greatest degradation according to:

$$
U_{\text {sens }}=\arg \max _{U_{k} \in U}\left\{d\left(U_{1}\right), \cdots, d\left(U_{N_{U}}\right)\right\}
$$

Thus, the user $U_{\text {sens }}$ is the most sensitive user in current assignment scheme. Accordingly, the optimal pilot sequence $\phi^{\mathrm{opt}}\left(U_{\text {sens }}\right)$ is then assigned to the most sensitive user $U_{\text {sens. }}$. Afterwards, it is required to remove the user $U_{\text {sens }}$ from the set $U$ and remove the pilot sequence $\phi^{\mathrm{opt}}\left(U_{\text {sens }}\right)$ from the available pilot sequence set $\Phi$

$$
\begin{aligned}
& U \leftarrow U \backslash U_{\text {sens }} \\
& \Phi \leftarrow \Phi \backslash \phi^{\text {opt }}\left(U_{\text {sens }}\right) .
\end{aligned}
$$

Also, the numbers of elements in set $U$ and $\Phi$ need to be updated as follows

$$
\begin{aligned}
& N_{U} \leftarrow N_{U}-1 \\
& N_{\Phi} \leftarrow N_{\Phi}-1
\end{aligned}
$$

These steps will be repeated until the set $U$ is empty, namely all the pilot sequences are allocated. We summarize the proposed pilot scheduling scheme in Table 1.

\subsection{Pilot scheduling scheme based on user grouping}

The aforementioned pilot scheduling scheme based on the degradation improves the system performance without increasing computational burden. However, users with poor channel condition would have performance bottleneck problem, causing communication interrupted.

\section{Table 1 The proposed scheme based on the degradation}

1: $\quad$ Get: $\beta_{i j k,} \quad j=1,2, \ldots, L, k=1,2, \ldots, K, U, \Phi ;$
2: $\quad$ While $N_{U} \neq 0$ do
3: $\quad$ For $k=1: N_{U}$
4: $\quad$ Calculate $\phi^{\text {opt }}\left(U_{k}\right)$ according to $(18)$
5: $\quad$ Calculate $\phi^{\text {sub }}\left(U_{k}\right)$ according to $(19)$
6: $\quad$ Calculate $d\left(U_{k}\right)$ according to $(20)$
7: $\quad$ End for
8: $\quad$ Calculate $U_{\text {sens }}$ according to $(21)$
9: $\quad$ Assign the optional pilot sequence $\phi^{\text {opt }}\left(U_{\text {sens }}\right)$ to the user $U_{\text {sens }}$
10: $\quad U \leftarrow U U_{\text {sens }}$
11: $\quad \Phi \leftarrow \Phi \backslash \phi^{\text {opt }}\left(U_{\text {sens }}\right)$
12: $\quad N_{U} \leftarrow N_{U}-1$
13: $\quad N_{\Phi} \leftarrow N_{\Phi}-1$
14: $\quad$ End while


Accordingly, a pilot scheduling scheme based on user grouping is proposed when an extra set of orthogonal pilot sequences exist, referring to the idea in [25].

\subsubsection{Basic idea}

The scheme, which is a tradeoff between system performance and pilot overhead, divides the users into the center user group and the edge user group according to different levels of pilot contamination based on the large-scale fading. The proposed scheme assigns orthogonal pilot sequences to the edge users, while the center users reuse the same pilot sequences.

\subsubsection{Pilot assignment}

As we know from (10), the uplink asymptotic SINR only relates to the large-scale fading of users who reuse the pilot sequences. Thus, we define the users' channel quality which is the square of their corresponding large-scale fading

$$
S_{i k}^{s i g}=\beta_{i i k}^{2}
$$

As a consequence, users are grouped according to the channel quality $S_{i k}^{s i g}$. Here, we can make an assumption that the number of edge users in each cell is equal and $K_{j e}$ denotes the number of edge users, $K_{j c}$ denotes the number of center users, namely $K_{c}=K_{j c}, K_{e}=K_{j e}$, and $j=1, \ldots, L$. Users with larger channel quality belong to the center user group, and they will reuse the same pilot sequences. On the other hand, users with poor channel quality belong to the edge user group, and they are assigned with orthogonal pilot sequences.

As we know, center users still suffer from pilot contamination; thus, their SINR are the same as (10). Differently, edge users do not suffer from pilot contamination because they are assigned with orthogonal pilot sequences. SINR of edge users goes to infinity when the number of BS antennas goes to infinity. However, the proposed pilot scheduling scheme based on user grouping increases the pilot overhead, which is calculated by

$$
\mu_{0} \times \frac{L K_{e}+K_{c}}{K} \leftarrow \mu_{0}
$$

where $K$ denotes the number of users in each cell, which is the number of orthogonal pilot sequence set when FRPS (fully reused pilot scheduling) scheme is adopted. $L K_{e}+$ $K_{c}$ denotes the number of orthogonal pilot sequence set when the proposed scheme is adopted.

The proposed scheme improves the QoS of edge users greatly, however, with a lack of flexibility. Therefore, an improved pilot scheduling scheme based on user grouping is proposed inspired by the study in [26]. The improved scheme employs the grouping parameter $\lambda$ to choose the edge users dynamically. Figure 2 describes the grouping parameter illustration of pilot scheduling scheme based

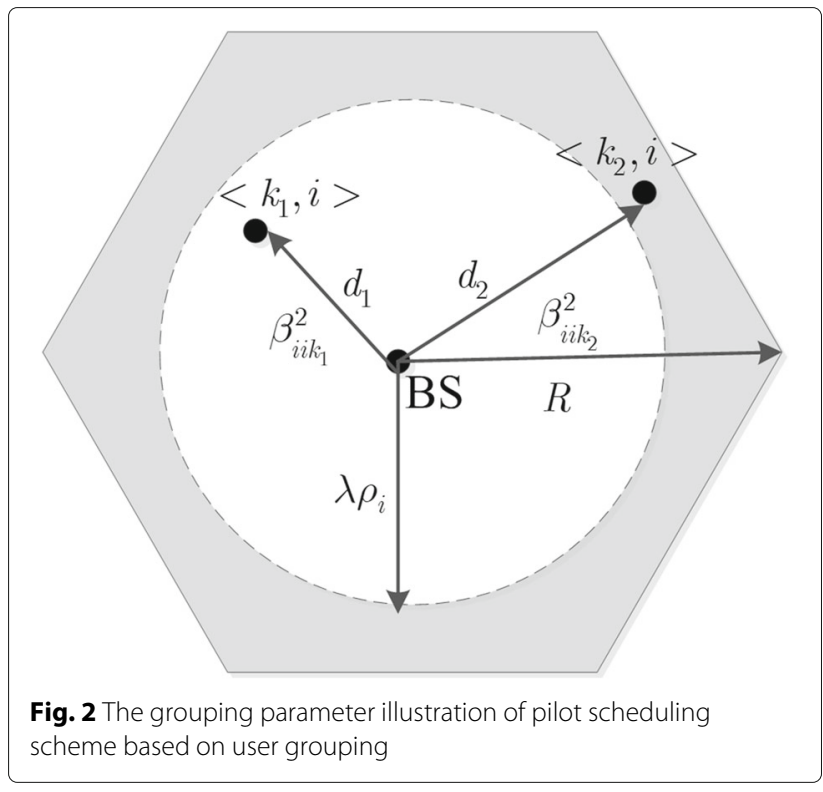

on user grouping, where the dashed area denotes edge area which meets the grouping requirements.

The improved scheme is able to improve the flexibility and accuracy simultaneously. When the grouping parameter $\lambda$ is fixed, according to the improved scheme based on large-scale fading coefficients, the grouping criterion can be represented by

$$
S_{i k}^{s i g} \geq \lambda \rho_{i} \rightarrow\left\{\begin{array}{l}
\text { Yes } \rightarrow \text { center users } \\
\text { No } \rightarrow \text { edge users }
\end{array},\right.
$$

where $\lambda \rho_{i}$ denotes the grouping threshold value. The main purpose of the improved scheme is to avoid that users with good channel conditions may be divided into edge user group or users with poor channel conditions are divided into center user group. As a result, we must take into consideration various factors including the user with best channel condition and the user with worst channel condition. Accordingly, $\rho_{i}$ can be written as

$\rho_{i}=\frac{\max \left\{S_{i 1}^{\mathrm{sig}}, S_{i 2}^{\mathrm{sig}}, \ldots, S_{i K}^{\mathrm{sig}}\right\}+\min \left\{S_{i 1}^{\mathrm{sig}}, S_{i 2}^{\mathrm{sig}}, \ldots, S_{i K}^{\mathrm{sig}}\right\}}{2}$.

As before, the improved pilot scheduling scheme based on user grouping incurs an extra pilot overhead, which is represented by

$$
\frac{\mu_{0}}{K}\left\{\sum_{j=1}^{L} K_{j e}+\max \left\{K_{1 c}, K_{2 c}, \cdots, k_{L c}\right\}\right\} \leftarrow \mu_{0} .
$$

To sum up, the proposed scheduling scheme based on the degradation assigns the optimal pilot sequence to the user who suffers from the greatest degradation greedily by computing the performance degradation of the users. 
Furthermore, a pilot scheduling scheme based on user grouping is proposed to optimize the scheme based on the degradation when an extra set of orthogonal pilot sequences is available. Under the improved pilot allocation method based on user grouping, the average uplink reachable rates of the center users and edge users are

$$
\begin{aligned}
C_{i k}^{u c}= & \left(1-\frac{\mu_{0}}{K}\left(\sum_{j=1}^{L} K_{j e}+\max \left\{K_{1 c}, K_{2 c}, \cdots, K_{L c}\right\}\right)\right) \\
& \mathrm{E}\left\{\log _{2}\left(1+S I N R_{i k}^{u c}\right)\right\} \\
C_{i k}^{u e}= & \left(1-\frac{\mu_{0}}{K}\left(\sum_{j=1}^{L} K_{j e}+\max \left\{K_{1 c}, K_{2 c}, \cdots, K_{L c}\right\}\right)\right) \\
& \mathrm{E}\left\{\log _{2}\left(1+S I N R_{i k}^{u e}\right)\right\}
\end{aligned}
$$

where $E\{\cdot\}$ denotes the expectation operation.

\section{Numerical results}

In this section, we investigate the performance of the scheme proposed in this paper through Monte-Carlo simulations. A typical hexagonal cellular network with $L$ cells is considered, where each cell has $K$ users with singleantenna and a BS with $M$ antennas. The center cell surrounded by other cells is considered as the target cell. The system parameters are summarized in Table 2.

Figure 3 draws the target cell's achievable sum rate when the number of BS antennas $M$ increases, where the results are obtained by 1000 independent simulation trials. It is evident that the proposed scheme outperforms the conventional approaches, for example, its gain over the RPS scheme is about $3.3 \mathrm{bps} / \mathrm{Hz}$ with $M=512$ and $K=10$. When the number of users in each cell $K=20$, the proposed scheme performs even better with a gain about $9.5 \mathrm{bps} / \mathrm{Hz}$.

Figure 4 plots the target cell's achievable sum rates of these three schemes when the shadowing fading increases

Table 2 Basic parameters of system simulation

\begin{tabular}{ll}
\hline Parameters & Values \\
\hline Number of cells $L$ & 7 \\
Cell radius $R$ & $500 \mathrm{~m}$ \\
Number of BS antennas $M$ & $16-1024$ \\
Number of users in each cell $K$ & {$[10,20]$} \\
Path loss $\alpha$ & 3.8 \\
Average SNR & $20 \mathrm{~dB}$ \\
Log normal shadowing fading $\sigma_{\text {shadow }}$ & $0-8 \mathrm{~dB}$ \\
Spectral efficiency $\mu_{0}$ & 0.05 \\
The pilot transmission power & $30 \mathrm{dBm}$ \\
The data transmission power & $30 \mathrm{dBm}$ \\
\hline
\end{tabular}

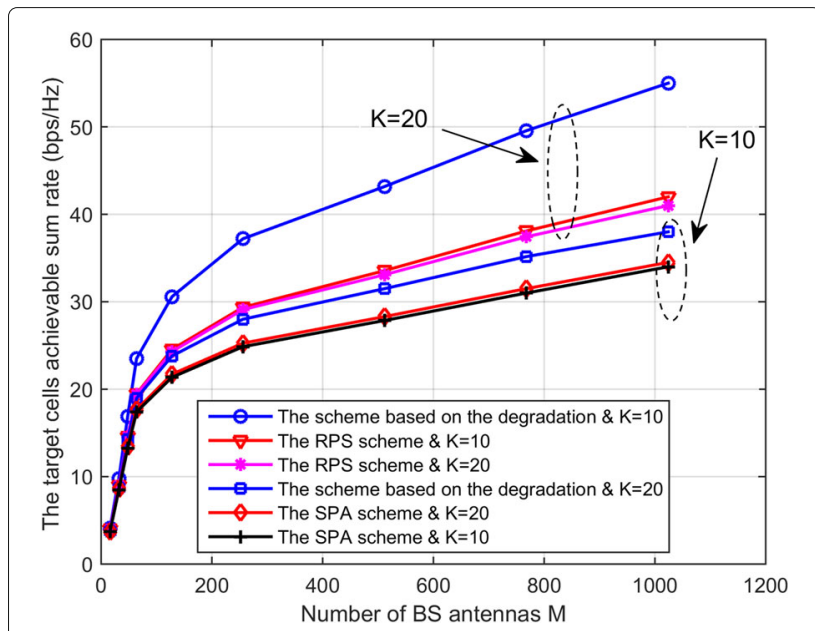

Fig. 3 The impact of the number BS antennas on the target cell's achievable sum rate

with $M=256$ and $K=10$. It is evident that as the shadowing fading increases, the target cell's achievable sum rate of conventional schemes declines continuously. In contrast, the rate of the proposed scheme is relatively stable at first and then grows slowly. This observation shows the robustness to the shadowing fading effect of the proposed algorithm.

Figure 5 demonstrates the SINR of edge users in target cell when the number of BS antennas $M$ increases, where the results are obtained with $K=10$ and $K_{j e}=1$, $j=1, \ldots, L$. For edge users, according to the result described by Fig. 5, we can find that the scheme based on user grouping outperforms the scheme based on degradation by about $7.6 \mathrm{~dB}$ with $M=256$. It is evident that the pilot contamination problem of edge users is reduced effectively by the strategy based on user grouping.

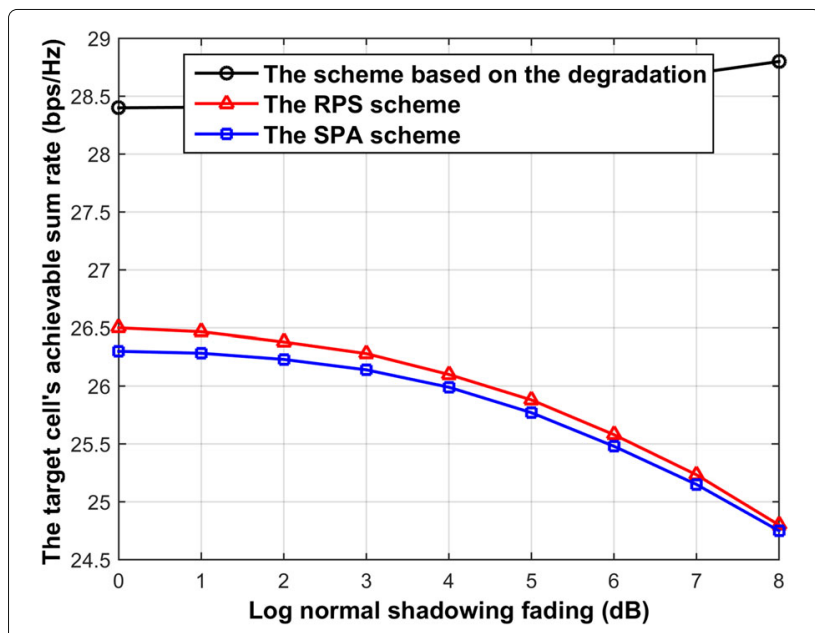

Fig. 4 The impact of shadowing fading on the target cell's achievable sum rate 


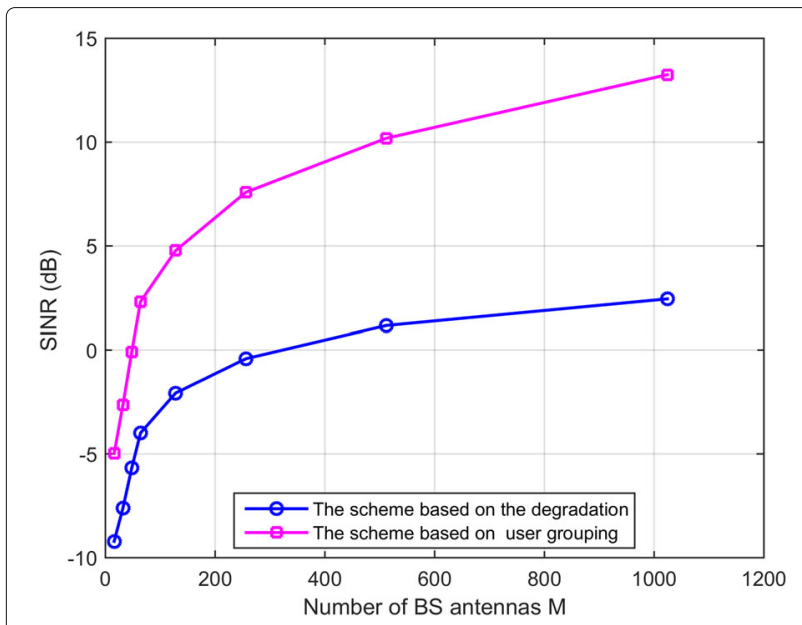

Fig. 5 The impact of the number of BS antennas on the SINR of edge users in target cell

Figure 6 plots the target cell's achievable sum rate when the number of edge users $K_{j e}$ increases with $M=512$ and $K=10$. From the result, it is readily observed that the scheme based on user grouping outperforms the scheme based on degradation when the number of edge users is less than 5 . When the number of edge users is larger than 5 , however, the cost of the pilot overhead cancels out the performance gain brought by user grouping. Therefore, careful attention needs to be paid when evaluating schemes based on user grouping and degradation.

Figure 7 shows the target cell's achievable sum rate when the grouping parameter $\lambda$ increases with $M=512$ and $K=10$. From the result, we can find that the improved scheme based on user grouping outperforms the scheme based on degradation when the grouping parameter $\lambda$ is larger than 0.5 . While if the grouping parameter $\lambda$ is less

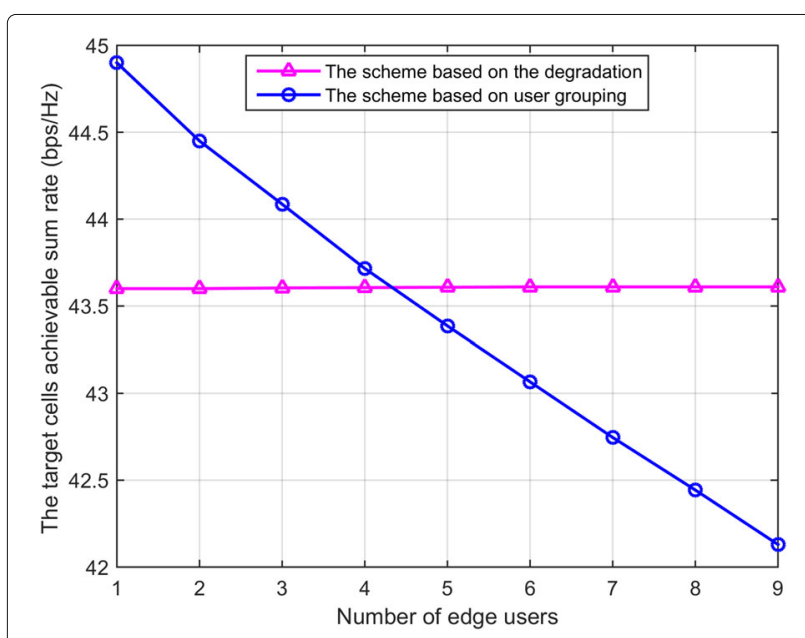

Fig. 6 The impact of the edge user number on the target cell's achievable sum rate

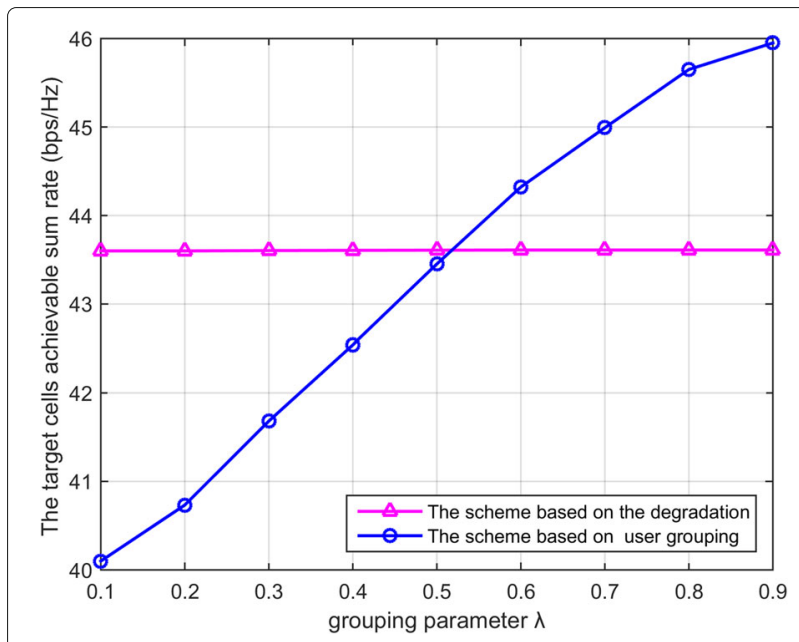

Fig. 7 The impact of grouping parameter on the target cell's achievable sum rate

than 0.5 , the cost of the pilot overhead cancels out the performance gain brought by user grouping. Comparing the results of Figs. 6 and 7, it can be concluded that the improved scheme is of more flexibility and accuracy. More importantly, Figs. 6 and 7 show that the scheme based on user grouping can improve the system performance effectively if a reasonable number of edge users or grouping parameter $\lambda$ is selected.

\section{Conclusions}

In this paper, a pilot scheduling scheme based on the degradation is proposed to maximize the target cell's achievable sum rate in massive MIMO systems. By exploiting the large-scale characteristics of fading channels, the proposed scheme assigns the optimal pilot sequence to the user who suffers from greatest degradation greedily. Because the users with poor channel condition have performance bottleneck problem which causes communication interrupted, a pilot scheduling scheme based on user grouping is proposed according to different levels of pilot contamination. The simulation results show that the proposed scheme based on degradation can reduce the impact of shadowing fading on the system performance and improve the target cell's achievable sum rate effectively. Furthermore, the pilot scheduling scheme based on user grouping not only solves the edge users' pilot contamination problem but also improves the system performance if a reasonable number of edge users or grouping parameter $\lambda$ is selected.

\section{Abbreviations}

AWGN: Additive white Gaussian noise; BS: Base station; CSI: Channel state information; D2D: Device-to-device; LS: Least square; Massive MIMO: Massive multi-input multi-output; MF: Match filter; QoS: Quality of service; RPS: Random pilot scheduling; SPA: Smart pilot assignment; SINR:

Signal-to-interference plus noise ratio 


\section{Acknowledgements}

The authors would like to thank Dr. Wenjiang Feng of Chongqing University for providing the code of the RPS and SPA algorithms.

\section{Funding}

This work is supported by the National Natural Science Foundation of China under grant 61201177 .

\section{Authors' contributions}

$\mathrm{YW}, \mathrm{TL}$, and $\mathrm{MC}$ contributed to the main idea, designed and implemented the algorithms, and drafted the manuscript. LL and WX designed and carried out the simulation and analyzed the results. All authors read and approved the final manuscript.

\section{Competing interests}

The authors declare that they have no competing interests.

\section{Publisher's Note}

Springer Nature remains neutral with regard to jurisdictional claims in published maps and institutional affiliations.

Received: 28 July 2017 Accepted: 11 January 2018

Published online: 22 January 2018

\section{References}

1. B Le, V Lau, E Jorswieck, N-D Dao, A Haghighat, DI Kim, T Le-Ngoc, Enabling $5 \mathrm{G}$ mobile wireless technologies. EURASIP J. Wirel. Commun. Netw. 2015(1), 1-14 (2015)

2. HR Wang, YH Wang, YM Huang, LX Yang, Pilot contamination reduction in very large MIMO cellular network. J. Commun. 29(2), 171-180 (2014)

3. J Zuo, J Zhang, C Yuen, W Jiang, W Luo, Energy-efficient downlink transmission for multicell massive DAS with pilot contamination. IEEE Trans. Veh. Technol. 66(2), 1209-1221 (2017)

4. TL Marzetta, Noncooperative cellular wireless with unlimited numbers of base station antennas. IEEE Trans. Wirel. Commun. 9(11), 3590-3600 (2010)

5. J Hoydis, S Ten Brink, M Debbah, Massive MIMO in the ul/dl of cellular networks: how many antennas do we need?. IEEE J. Sel. Areas Commun. 31(2), 160-171 (2013)

6. J Jose, A Ashikhmin, TL Marzetta, S Vishwanath. Pilot contamination and precoding in multi-cell TDD systems, vol. 10, (2011), pp. 2640-2651

7. H Yin, D Gesbert, M Filippou, Y Liu. A coordinated approach to channel estimation in large-scale multiple-antenna systems, vol. 31, (2013), pp. 264-273

8. F Fernandes, A Ashikhmin, TL Marzetta. IEEE J. Sel. Areas Commun, vol. 31, (2013), pp. 192-201

9. H Holma, S Heikkinen, OA Lehtinen, A Toskala. Interference considerations for the time division duplex mode of the UMTS terrestrial radio access, vol. 18, (2000), pp. 1386-1393

10. CK Wen, S Jin, KK Wong, JC Chen, P Ting, Channel estimation for massive MIMO using Gaussian-mixture Bayesian learning. IEEE Trans. Wirel. Commun. 14(3), 1356-1368 (2015)

11. S Nguyen, A Ghrayeb, in Wireless Communications and Networking Conference (WCNC), 2013 IEEE. Compressive sensing-based channel estimation for massive multiuser MIMO systems (IEEE, Shanghai, 2013), pp. $2890-2895$

12. J Zuo, J Zhang, C Yuen, W Jiang, W Luo. Multicell multiuser massive MIMO transmission with downlink training and pilot contamination precoding, vol. 65, (2016), pp. 6301-6314

13. J Zuo, J Zhang, C Yuen, W Jiang, W Luo. Energy efficient user association for cloud radio access networks, vol. 4, (2016), pp. 2429-2438

14. WA Mahyiddin, PA Martin, PJ Smith, in Vehicular Technology Conference (VTC Fall), 2014 IEEE 80th. Pilot contamination reduction using time-shifted pilots in finite massive MIMO systems (IEEE, Vancouver, 2014), pp. 1-5

15. HR Wang, YH Wang, YM Huang, LX Yang, Pilot contamination reduction in very large MIMO multi-cell TDD systems. J. Signal Process. (Xinhao Chuli). 29(2), 171-180 (2013)

16. V Saxena, G Saxena, E Karipidis, in Vehicular Technology Conference (VTC), 2015. Mitigating pilot contamination by pilot reuse and power control schemes for massive MIMO systems (IEEE, Glasgow, 2015), pp. 1-6
17. HXu, N Huang, Z Yang, Pilot allocation and power control in D2D underlay massive MIMO systems. IEEE Commun. Lett. 21(1), 112-115 (2017)

18. M Liu, X Chen, $\mathrm{W} X u$, in International Conference on Wireless Communications \& Signal Processing. Grouped pilot reuse for channel estimation in massive MIMO networks (IEEE, Chiang Mai, 2016), pp. 1-5

19. Z Zhao, Z Chen, Y Liu, in Wireless Telecommunications Symposium (WTS), 2015. Cell sectorization-based pilot assignment scheme in massive mimo systems (IEEE, 2015), pp. 1-5

20. X Zhu, Z Wang, C Qian, L Dai, J Chen, S Chen, L Hanzo, Soft pilot reuse and multicell block diagonalization precoding for massive mimo systems. IEEE Trans. Veh. Technol. 65(5), 3285-3298 (2016)

21. H Yin, D Gesbert, M Filippou, Y Liu, A coordinated approach to channel estimation in large-scale multiple-antenna systems. IEEE J. Sel. Areas Commun. 31(2), 264-273 (2013)

22. X Zhu, Z Wang, L Dai, C Qian, Smart pilot assignment for massive MIMO. IEEE Commun. Lett. 19(9), 1644-1647 (2015)

23. M Li, S Jin, X Gao, in Wireless Communications \& Signal Processing (WCSP), 2013 International Conference On. Spatial orthogonality-based pilot reuse for multi-cell massive MIMO transmission (IEEE, Hangzhou, 2013), pp. 1-6

24. AM Girgis, B Abdelhamid, S Elramly, in Wireless Communications and Networking Conference Workshops. Improved pilot sequences allocation in massive MIMO systems (IEEE, San Francisco, 2017), pp. 1-6

25. H-B Chang, I Rubin, Optimal downlink and uplink fractional frequency reuse in cellular wireless networks. IEEE Trans. Veh. Technol. 65(4), 2295-2308 (2016)

26. $X$ Yang, A multilevel soft frequency reuse technique for wireless communication systems. IEEE Commun. Lett. 18(11), 1983-1986 (2014)

\section{Submit your manuscript to a SpringerOpen ${ }^{\circ}$ journal and benefit from:}

- Convenient online submission

- Rigorous peer review

- Open access: articles freely available online

- High visibility within the field

- Retaining the copyright to your article

Submit your next manuscript at $>$ springeropen.com 